\title{
Preparation and Photocatalytic Water Splitting Hydrogen Production of Titanium Dioxide Nanosheets
}

\author{
Fuying Li $\mathbb{D},{ }^{1,2}$ Yin Huang, ${ }^{1}$ Hongling Peng, ${ }^{1}$ Yu Cao, ${ }^{1}$ and Yu Niu $\mathbb{D}^{1,2}$ \\ ${ }^{1}$ College of Resources and Chemical Engineering, Sanming University, Sanming 365004, China \\ ${ }^{2}$ Collaborative Innovation Center of 2011 Clean Coal Gasification Technology, Sanming 365004, China \\ Correspondence should be addressed to Yu Niu; niuyu200704@163.com
}

Received 27 December 2019; Revised 29 February 2020; Accepted 16 March 2020; Published 5 August 2020

Academic Editor: Chun Chang

Copyright ( 2020 Fuying Li et al. This is an open access article distributed under the Creative Commons Attribution License, which permits unrestricted use, distribution, and reproduction in any medium, provided the original work is properly cited.

\begin{abstract}
Improving the efficiency of photocatalytic water splitting to produce hydrogen is currently a hot topic in research. $\mathrm{TiO}_{2}$ nanosheets are a good carrier of photocatalytic materials and have become attractive materials in the new century because of their high active surface exposure characteristics and special morphology. Considering the advantages and disadvantages of conventional chemical and physical methods that are used for preparing $\mathrm{TiO}_{2}$ nanosheets, an optimized scheme for the preparation of $\mathrm{TiO}_{2}$ nanosheets via hydrothermal calcination was proposed. X-ray powder diffraction (XRD), scanning electron microscopy (SEM), and UV-visible diffuse reflection absorption spectra (DRS) were used to characterize the structure and morphology of the $\mathrm{TiO}_{2}$ nanosheets, and differences in the photocatalytic water splitting hydrogen production activity of the different calcination temperatures were compared. The suitable calcination temperature of the $\mathrm{TiO}_{2}$ nanosheets was $400^{\circ} \mathrm{C}$, and the hydrogen production rate was $270 \mu \mathrm{mol} / \mathrm{h}$, which indicated that the sheet structure was beneficial for improving the photocatalytic water splitting hydrogen production performance of the material. It is hoped that this work will support the regulation of the surface morphology and surface modification of nanomaterials.
\end{abstract}

\section{Introduction}

Negative effects of the rapid development of science and technology include deterioration of the ecological environment and depletion of the earth's resources. This has caused humanity to face two major problems, environmental pollution and an energy crisis. Renewable clean energy is undoubtedly an effective way to simultaneously solve environmental and energy problems. Since the 21st century, research on a variety of alternative types of energy (solar, biomass, water, wind, geothermal, tidal, hydrogen, and biogas) has been conducted. Great efforts have been made in this area of research [1]. Among the kinds of alternative energy, hydrogen energy has excellent characteristics, such as high energy, a wide combustion range, and good thermal conductivity. Hydrogen energy exists in three forms (gas, liquid, and solid), which is convenient for storage and transportation. The hydrogen combustion process and products are nonpolluting, and hydrogen is recognized as the most popular clean energy in the world. Hydrogen can be produced by decomposing the most abundant water resources on earth, and thus, hydrogen energy is known as "the oil of the future" $[2,3]$. However, the current production of hydrogen is mainly from burning fossil fuels or a high-energy consumption process, which is contrary to the environmentally friendly and sustainable development that people desire. In 1972, Fujishima and Honda discovered that water could be decomposed to produce hydrogen via the irradiation of a titanium dioxide electrode with sunlight; this brought the photocatalytic decomposition of hydrogen to produce hydrogen technology into the spotlight [4]. In the following decades, researchers have engaged in many discussions regarding the research and development of semiconductor materials and their mechanism of action, and this has promoted photocatalytic technology as a promising new method for producing hydrogen [5]. At present, most of the semiconductor photocatalytic materials that have been discovered are $n$-type semiconductors, such as $\mathrm{TiO}_{2}, \mathrm{ZnS}, \mathrm{ZnO}, \mathrm{WO}_{3}, \mathrm{SnO}_{2}$, CdS, and $\mathrm{Fe}_{2} \mathrm{O}_{3}$. Among them, 
$\mathrm{TiO}_{2}$ is promising because of its strong oxidizing power and cheap availability and because it is nontoxic and harmless. It also has many advantages, such as being not easily prone to photocorrosion, and these advantages have been widely studied and used [6-12].

In recent years, semiconductor materials with nanostructures have shown significant advantages in terms of magnetic, superconductivity, optical, and thermodynamic properties $[13,14]$. Therefore, the physical and chemical properties of $\mathrm{TiO}_{2}$ nanomaterials are different from those of conventional $\mathrm{TiO}_{2}$ materials. Among the differences, $\mathrm{TiO}_{2}$ nanosheets provide more active centers, have a larger specific surface area than nanorods, and have better high active surface exposure than nanotubes, showing better catalytic performance [15-19]. The conventional methods for preparing $\mathrm{TiO}_{2}$ nanosheets can be divided into two methods: physical methods (vapor condensation method, high-energy ball milling method, etc.) and chemical methods (sol-gel, liquid deposition, microemulsion, hydrothermal calcination, etc.). Nanosheets that are prepared via a simple method often have a disordered arrangement, and nanosheets that are produced via a complicated method have a low yield. Therefore, from a comprehensive analysis of the advantages and disadvantages of the common methods, an optimization scheme for preparing $\mathrm{TiO}_{2}$ nanosheets with controlled morphology prepared via a hydrothermal calcining method [20-22] was proposed.

\section{Experimental Part}

2.1. Preparation of $\mathrm{TiO}_{2}$ Nanosheets. The preparation process of $\mathrm{TiO}_{2}$ nanosheets via hydrothermal calcination is shown as follows: At room temperature, $25 \mathrm{~mL}$ of tetrabutyl titanate and $2 \mathrm{~mL}$ of hydrofluoric acid were measured and mixed to obtain a transparent yellow solution. After ultrasonic treatment for $20 \mathrm{~min}$, the mixtures were magnetically stirred for $20 \mathrm{~min}$. Full contact was made with the hydrofluoric acid. The treated liquid was transferred to a hydrothermal reactor with a polytetrafluoroethylene liner, and the reactor was placed in a $180^{\circ} \mathrm{C}$ constant temperature incubator for hydrothermal treatment for $24 \mathrm{~h}$. The reactor was allowed to cool naturally to room temperature, and then, it was centrifuged to isolate a white precipitate. To remove the hydrofluoric acid remaining on the white precipitate, the precipitate was placed in a suitable amount of deionized water and magnetically stirred for a period of time. After centrifugation, the supernatant was removed, and the above operation was repeated until the $\mathrm{pH}$ value of the supernatant obtained via centrifugation was $\approx 7$. To remove the remaining fat-soluble substances on the white precipitate, the precipitate was magnetically stirred in an appropriate amount of absolute ethanol for a period of time. To convert the fluorinated $\mathrm{TiO}_{2}$ nanosheets to fluorine-free $\mathrm{TiO}_{2}$ nanosheets, $0.1 \mathrm{~mol} / \mathrm{L}$ of $\mathrm{NaOH}$ solution was used under magnetic stirring and then washed thoroughly with deionized water to remove the residual $\mathrm{NaOH}$. The white precipitate was dried under a constant temperature of $80^{\circ} \mathrm{C}$ for $6 \mathrm{~h}$ to obtain the $\mathrm{H}_{2} \mathrm{Ti}_{3} \mathrm{O}_{7} \cdot n \mathrm{H}_{2} \mathrm{O}$ nanosheets. Finally, a resistance box was used for calcinations at different temperatures $\left(300^{\circ} \mathrm{C}, 400^{\circ} \mathrm{C}\right.$, and $\left.500^{\circ} \mathrm{C}(2 \mathrm{~h})\right)$. The nanosheets were converted to anatase $\mathrm{TiO}_{2}$.
2.2. Characterization of Samples. A Bruker D8 Advance X-ray powder diffractometer was used to analyze the crystal phase structure of the catalyst sample. A ZEISS SIGMA scanning electron microscope was used to observe the surface morphology. A Varian Cary 500 UV-visible diffuse reflection absorption spectrometer was used to measure the light absorption performance, and the DRS spectrum was observed.

\subsection{Hydrogen Production Performance Test of Water} Photolysis. Photocatalytic performance was evaluated using a vacuum glass circulation system, as shown in Figure 1. Deionized water $(155 \mathrm{~mL})$ and the catalyst sample $(100 \mathrm{mg})$ were added to the photocatalytic reactor $(250 \mathrm{~mL})$ before the reaction. A sacrificial agent $(5 \mathrm{~mL}$ of methanol) was added under magnetic stirring, and then, the reactor was connected to the system. A chiller was used to maintain the temperature of the reaction solution at $10^{\circ} \mathrm{C}$ to avoid thermal effects, and then, the entire reaction system was evacuated using a vacuum pump. An ultraviolet lamp was used as a light source, and an online sample was taken every half hour under an argon atmosphere. A gas chromatograph was used to measure the peak value of the hydrogen response signal, which was converted to the amount of hydrogen.

\section{Results and Discussion}

3.1. Crystal Phase of the Sample. Figure 2 shows an XRD spectrum of the $\mathrm{TiO}_{2}$ nanosheets that were prepared at different calcination temperatures. As seen in the figure, the sample that was calcined at $300^{\circ} \mathrm{C}$ only showed the outline of the anatase phase. Also, the broad band between $2 \theta$ of $27^{\circ}$ and $30^{\circ}$, which is neither anatase phase nor rutile, was more obvious. The broad diffraction peak of the phase indicates that the hydrate $\mathrm{H}_{2} \mathrm{Ti}_{3} \mathrm{O}_{7} \cdot n \mathrm{H}_{2} \mathrm{O}$ was still present in the sample after calcination at $300^{\circ} \mathrm{C}$. The above observation indicates that the sample gradually changed to anatase $\mathrm{TiO}_{2}$ at this temperature, but the crystal effect was relatively poor. The prepared samples showed good crystal peaks that were characteristic of anatase. Also, the broad band between $2 \theta$ of $27^{\circ}$ and $30^{\circ}$ disappeared, and the double peak of $2 \theta$ at about $70^{\circ}$ also appeared. These observations indicate that after forging at $400^{\circ} \mathrm{C}$, there was no hydrate in the fired sample and it had been converted into anatase $\mathrm{TiO}_{2}$. The peaks characteristic of anatase for the sample that was calcined at $500^{\circ} \mathrm{C}$ are $2 \theta=25.1^{\circ}, 37.6^{\circ}, 48.0^{\circ}, 53.8^{\circ}, 55.0^{\circ}$, and $62.7^{\circ}$ (A, JCPDS 21-1272) all appeared. These indicate that the crystal form of the prepared $\mathrm{TiO}_{2}$ nanosheets was the most complete at this calcination temperature, consistent with previous studies [23-25].

3.2. Morphology of the Sample. Figure 3 shows the SEM images of the prepared $\mathrm{TiO}_{2}$ nanosheets at different calcination temperatures. Observations from the SEM images of the morphology and structure of the samples [26, 27] showed that the morphologies of the samples that were prepared at different calcination temperatures were significantly different. As seen in Figure 3(a), the sample that was calcined at $300^{\circ} \mathrm{C}$ has a granular agglomerate and does not show a sheet structure. Figure 3(b) shows that the sample calcined at 


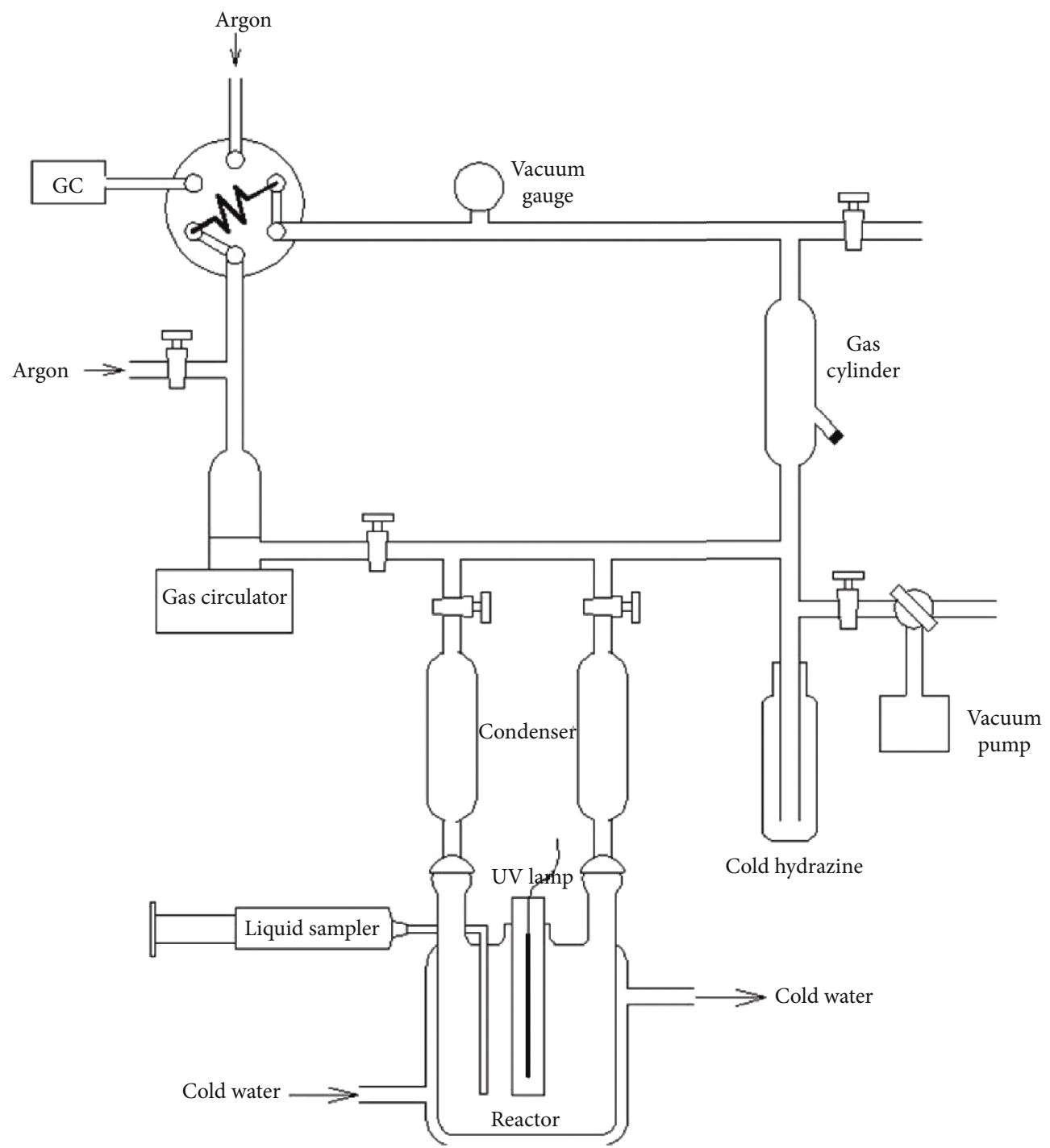

FIGURE 1: Vacuum glass circulation system for photocatalytic performance evaluation.

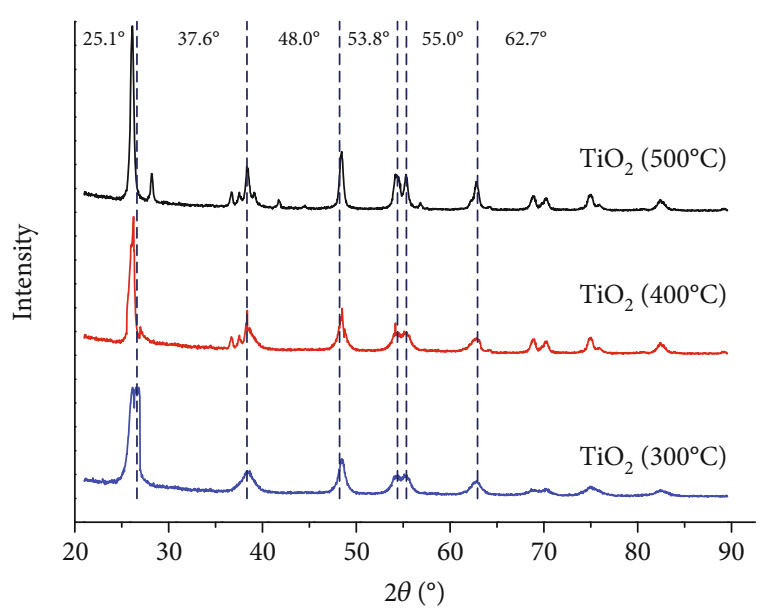

FIGURE 2: XRD spectra of samples calcined at different temperatures. $400^{\circ} \mathrm{C}$ has a sheet structure. The structure and shape were relatively regular. As seen in Figure 3(c), the sheet structure of the sample calcined at $500^{\circ} \mathrm{C}$ disappeared and was transformed into a block structure. These observations show that the suitable calcination temperature for preparing $\mathrm{TiO}_{2}$ with lamellar structure was $400^{\circ} \mathrm{C}$.

3.3. Spectral Absorption Properties of the Sample. Figure 4 shows the ultraviolet-visible diffuse reflection absorption spectra of the $\mathrm{TiO}_{2}$ nanosheets prepared at different calcination temperatures. $\mathrm{TiO}_{2}$ is an $n$-type semiconductor material. The light absorption threshold of anatase $\mathrm{TiO}_{2}$ is $380 \mathrm{~nm}$, and the corresponding forbidden bandwidth $(E g)$ is $3.2 \mathrm{eV}$. The straight line segment of the DRS spectrum is extended to intersect the abscissa axis, and the intersection point is the absorption wavelength threshold $(\lambda g)$. The band gap can be obtained from $E g=1240 / \lambda g$ [28]. As seen in Figure 4, although the sample shows a weak redshift in the UV absorption band at $400 \mathrm{~nm}$, it is clear that the absorption wavelength threshold exhibits an order of $\mathrm{TiO}_{2}\left(300^{\circ} \mathrm{C}\right)<\mathrm{TiO}_{2} \quad\left(500^{\circ} \mathrm{C}\right)<\mathrm{TiO}_{2}$ 

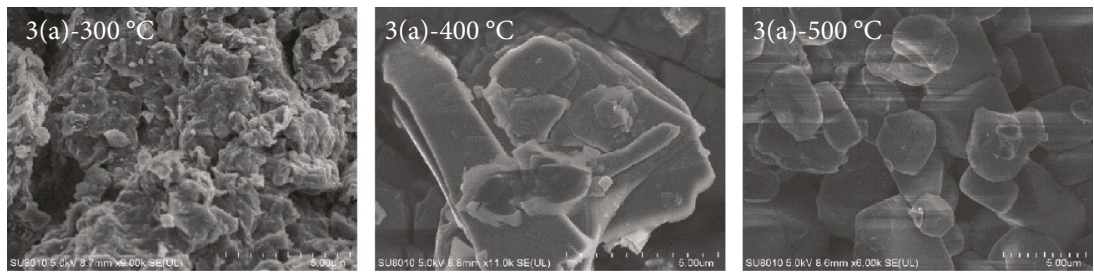

FIGURE 3: SEM images of samples at different calcination temperatures.

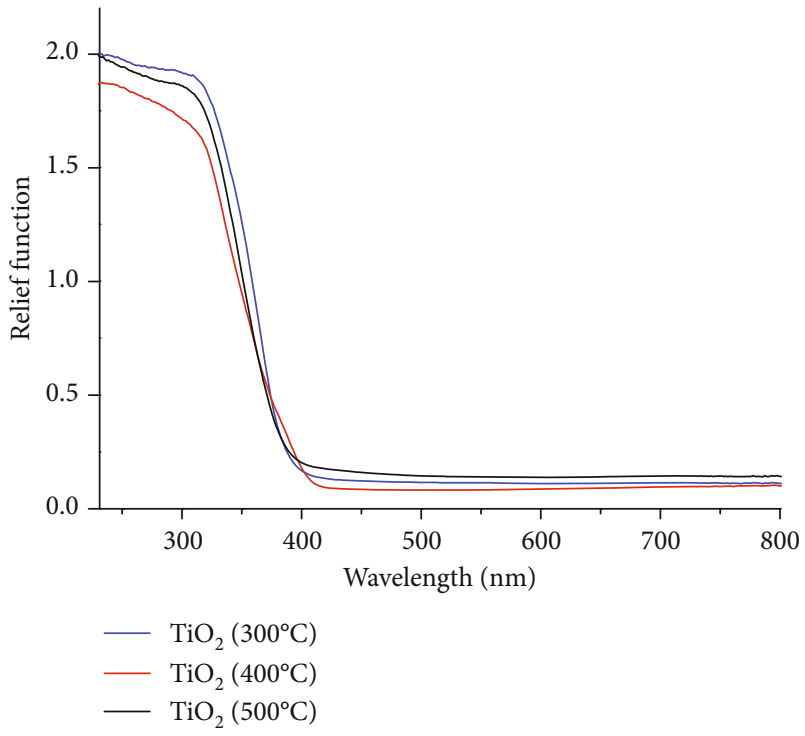

Figure 4: UV-visible diffuse reflection absorption spectrum of the sample.

$\left(400^{\circ} \mathrm{C}\right)$, indicating that the band gap of the sample that was prepared via calcination at $400^{\circ} \mathrm{C}$ had the smallest width and relatively high light absorption capacity [29].

3.4. Activity Comparison of Samples. The photocatalytic hydrogen splitting hydrogen production performance of the samples was tested as described in Section 2.3. A comparison of the photocatalytic hydrogen splitting hydrogen production activity is shown in Figure 5. The hydrogen production efficiency of the sample calcined at $400^{\circ} \mathrm{C}$ was the highest. The hydrogen production reached $1.35 \mathrm{mmol}$ in $5 \mathrm{~h}$, and this was calculated as $270 \mu \mathrm{mol} / \mathrm{h}$. For the hydrogen production efficiency of the samples calcined at $300^{\circ} \mathrm{C}$ and $500^{\circ} \mathrm{C}$, the values were only $58 \mu \mathrm{mol} / \mathrm{h}$ and $62 \mu \mathrm{mol} / \mathrm{h}$, respectively, which were even less than the photocatalytic hydrogen production efficiency of granular nano-P25 (80\% anataseand $20 \%$ rutile-mixed $\mathrm{TiO}_{2}$ ), which is $76 \mu \mathrm{mol} / \mathrm{h}$ [30].

Analysis of the combined XRD, SEM, and DRS characterization results showed that the complete anatase crystal form lamellar structure was conducive for improving the photocatalytic water and hydrogen production performance of the $\mathrm{TiO}_{2}$ samples. Compared to the lamellar structure, the granular aggregates contained hydrates and bulk anatase crystal phase $\mathrm{TiO}_{2}$. These are not conducive to the production of hydrogen from the photolysis of water, and this may be because the granular and massive morphology greatly

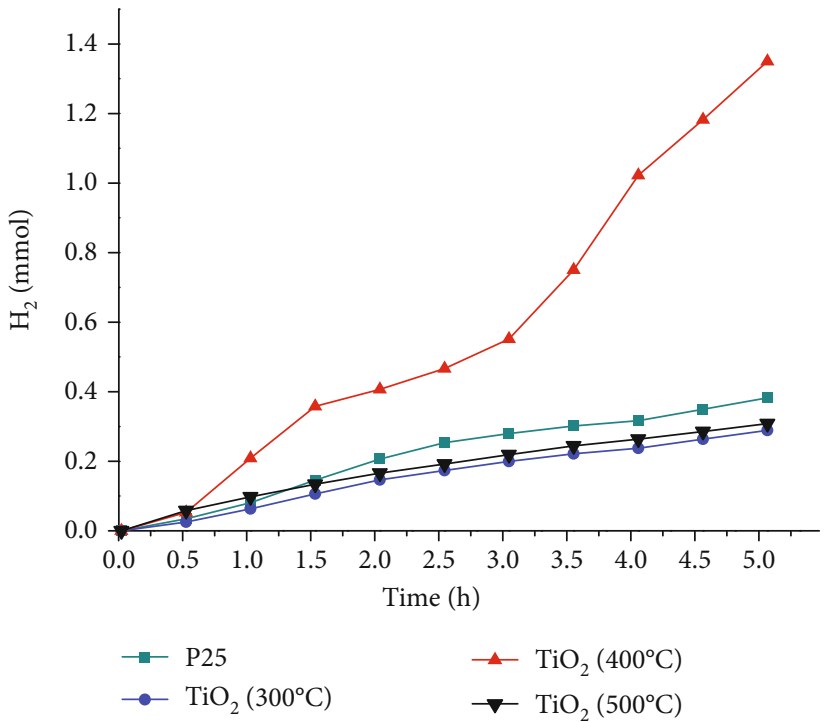

Figure 5: Photocatalytic decomposition of water to produce hydrogen.

reduces the specific surface area of the sample and blocks the active surface $[31,32]$.

The multicycle performance of the samples calcined at $400^{\circ} \mathrm{C}$ is shown in Figure 6. After three cycles, the $\mathrm{TiO}_{2}$ $\left(400^{\circ} \mathrm{C}\right)$ sample still maintained good photohydrolytic hydrogen production activity, indicating that the sample had good photocorrosion resistance and sustained stability. After recycling, the hydrogen production efficiency of the photolyzed water was lower than that of the previous cycle, and this was because of loss during the sample recovery.

\section{Conclusions}

To optimize the hydrothermal calcination method, samples were prepared at calcination temperatures of $300^{\circ} \mathrm{C}, 400^{\circ} \mathrm{C}$, and $500^{\circ} \mathrm{C}$, and the samples were characterized via $\mathrm{XRD}$, SEM, and DRS. The hydrogen production activity of the photocatalytic decomposition of water was also tested, and the experimental results were analyzed. An appropriate calcination temperature was selected. $\mathrm{TiO}_{2}$ calcined at $300^{\circ} \mathrm{C}$ had highly agglomerated particles with poor crystal quality that were partly in the form of hydrate $\left(\mathrm{H}_{2} \mathrm{Ti}_{3} \mathrm{O}_{7} \cdot n \mathrm{H}_{2} \mathrm{O}\right)$, and the hydrogen production efficiency of the photolysis of water was low. $\mathrm{TiO}_{2}$ calcined at $500^{\circ} \mathrm{C}$ had a block structure, and this may have been because of the high temperature of the calcination. This resulted in a large reduction in the specific 


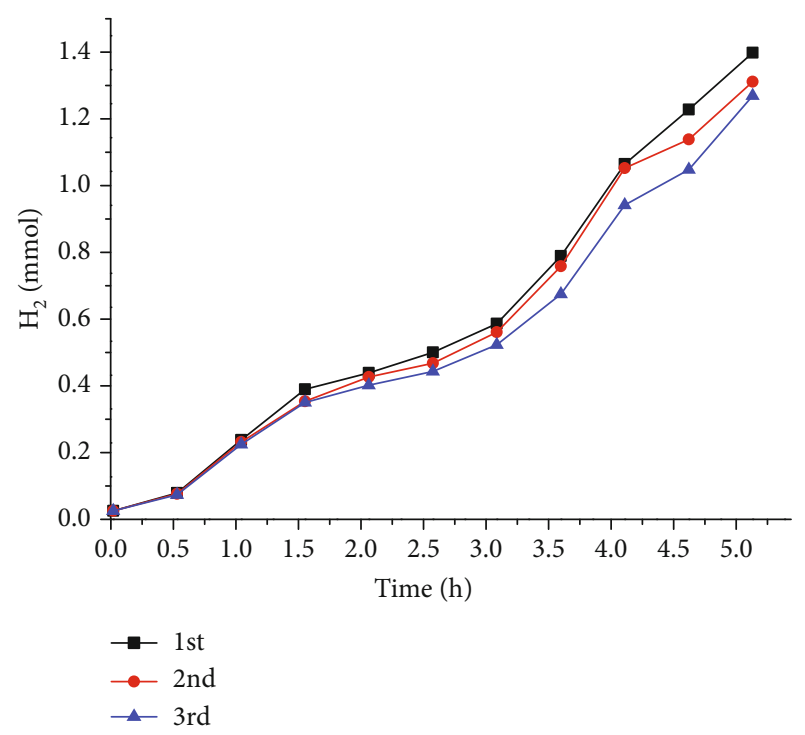

Figure 6: Performance of the sample after multiple cycles.

surface area and greatly reduced the catalytic capacity. At a calcination temperature of $400^{\circ} \mathrm{C}, \mathrm{TiO}_{2}$ nanosheets with complete crystals, uniform grain distribution, and controllable morphology were prepared. The nanosheets had excellent photohydrolytic hydrogen production activity, and this may have been because of the increase in the specific surface area as a result of the lamellar structure morphology and the characteristics of the highly active exposed surface.

\section{Data Availability}

All data included in this study are available upon request by contacting the corresponding author.

\section{Conflicts of Interest}

The authors declare no conflicts of interest regarding the publication of this paper.

\section{Acknowledgments}

This study received financial support from the Outstanding Youth Program Foundation of Fujian (No. 201752), the Key Project Young Natural Science of Fujian Provincial University (No. JZ160478), the Natural Science Foundation of Fujian Province (No. 2019J01818), the Resources and Environment Monitoring and Sustainable Management and Utilization of Fujian Provincial Key Laboratory (No. ZD1804), the University Student Innovation of Fujian (No. 201711311036), and the Collaborative Innovation Center of Clean Coal Gasification Technology of Fujian (Nos. XK1401 and XK1403).

\section{References}

[1] R. Miandad, M. Rehan, O. K. M. Ouda et al., "Waste-to-hydrogen energy in Saudi Arabia: challenges and perspectives," in Biohydrogen Production: Sustainability of Current Technology and Future Perspective, pp. 237-252, Springer, 2017.
[2] B. Zohuri, "Hydrogen energy technology, renewable source of energy," in Hybrid Energy Systems, pp. 135-179, Springer, Cham, 2018.

[3] J. Ren, D. Xu, H. Cao, S.'. Wei, L. Dong, and M. E. Goodsite, "Sustainability Decision Support Framework for the Prioritization of Hydrogen Energy Systems," in Hydrogen Economy, pp. 225-276, Springer, 2017.

[4] A. Fujishima and K. Honda, "Electrochemical photolysis of water at a semiconductor electrode," Nature, vol. 238, no. 5358, pp. 37-38, 1972.

[5] E. Zhang, "Photocatalytic hydrogen evolution from water splitting using semiconductors: advance, challenge and prospects," Chinese Journal of Inorganic Chemistry, vol. 33, no. 2, pp. 177-209, 2017.

[6] W. Liu, Y. Xu, W. Zhou et al., "A facile synthesis of hierarchically porous $\mathrm{TiO}_{2}$ microspheres with carbonaceous species for visible-light photocatalysis," Journal of Materials Science \& Technology, vol. 33, no. 1, pp. 39-46, 2017.

[7] V. K. Gupta, A. Fakhri, M. Azad, and S. Agarwal, "Synthesis and characterization of $\mathrm{Ag}$ doped $\mathrm{ZnS}$ quantum dots for enhanced photocatalysis of Strychnine as a poison: charge transfer behavior study by electrochemical impedance and time-resolved photoluminescence spectroscopy," Journal of Colloid and Interface Science, vol. 510, pp. 95-102, 2018.

[8] M. Baek, E. J. Kim, S. W. Hong, W. Kim, and K. Yong, "Environmentally benign synthesis of $\mathrm{CuInS}_{2} / \mathrm{ZnO}$ heteronanorods: visible light activated photocatalysis of organic pollutant/bacteria and study of its mechanism," Photochemical and Photobiological Sciences, vol. 16, no. 12, pp. 1792-1800, 2017.

[9] X. Zeng, Z. Wang, G. Wang et al., "Highly dispersed $\mathrm{TiO}_{2}$ nanocrystals and $\mathrm{WO}_{3}$ nanorods on reduced graphene oxide: Z-scheme photocatalysis system for accelerated photocatalytic water disinfection," Applied Catalysis B: Environmental, vol. 218, pp. 163-173, 2017.

[10] L. Peng, Y. Xiao, X. L. Wang, D. W. Feng, H. Yu, and X. T. Dong, "Realization of visible light photocatalysis by wide band gap pure $\mathrm{SnO}_{2}$ and study of $\mathrm{In}_{2} \mathrm{O}_{3}$ Sensitization porous $\mathrm{SnO}_{2}$ Photolysis catalyst," ChemistrySelect, vol. 4, no. 29, pp. 8460 8469, 2019.

[11] A. Singh and A. S. K. Sinha, "Synthesis and characterization of CdS-based ternary composite for enhanced visible light-driven photocatalysis," Journal of Physics and Chemistry of Solids, vol. 120, pp. 123-132, 2018.

[12] Q. Du and Q. Gao, "Template-free synthesis of mesoporous $\alpha$ Fe2O3nanoflowers with short charge-carrier diffuse distance for superior photocatalysis," Materials Technology, vol. 32, no. 12, pp. 724-728, 2017.

[13] N. H. Karam, N. R. Jber, and A. H. al-Dujaili, "A new series of triazine-core based mesogenic derivatives: synthesis, characterization and mesomorphic study," Molecular Crystals and Liquid Crystals, vol. 675, no. 1, pp. 39-48, 2019.

[14] J. Liu, J. Feng, J. Gui et al., "Metal@semiconductor core-shell nanocrystals with atomically organized interfaces for efficient hot electron-mediated photocatalysis," Nano Energy, vol. 48, pp. 44-52, 2018.

[15] I. Daou, N. Moukrad, O. Zegaoui, and F. Rhazi Filali, "Antimicrobial activity of $\mathrm{ZnO}-\mathrm{TiO}_{2}$ nanomaterials synthesized from three different precursors of $\mathrm{ZnO}$ : influence of $\mathrm{ZnO} / \mathrm{TiO}_{2}$ weight ratio," Water Science and Technology, vol. 77, no. 5, pp. 1238-1249, 2018. 
[16] C. Chen, X. Xin, J. Zhang et al., "Few-layered $\mathrm{MoS}_{2}$ Nanoparticles loaded $\mathrm{TiO}_{2}$ Nanosheets with Exposed $\{001\}$ Facets for enhanced photocatalytic activity," Nano, vol. 13, no. 11, article 1850129, 2018.

[17] L. Ma, Y. Zhu, X. Li, C. Yang, P. Han, and G. Song, "The architecture of carbon fiber- $\mathrm{TiO}_{2}$ nanorods hybrid structure in supercritical water for reinforcing interfacial and impact properties of CF/epoxy composites," Polymer Testing, vol. 66, pp. 213-220, 2018.

[18] L. Zhong, X. Li, R. Liu, X. Wei, and J. Li, “A visible-light-driven photoelectrochemical molecularly imprinted sensor based on titanium dioxide nanotube arrays loaded with silver iodide nanoparticles for the sensitive detection of benzoyl peroxide," The Analyst, vol. 144, no. 10, pp. 3405-3413, 2019.

[19] H. Fu, L. Yang, D. Hu et al., "Titanium dioxide nanoheterostructure with nanoparticles decorating nanowires for high-performance photocatalysis," International Journal of Hydrogen Energy, vol. 43, no. 22, pp. 10359-10367, 2018.

[20] Y. Zhao, Y. Zhao, R. Shi et al., "Tuning oxygen vacancies in ultrathin $\mathrm{TiO}_{2}$ Nanosheets to boost photocatalytic nitrogen fixation up to $700 \mathrm{~nm}$," Advanced Materials, vol. 31, no. 16, article 1806482, 2019.

[21] T. Shi, Y. Duan, K. Lv et al., "Photocatalytic oxidation of acetone over high thermally stable $\mathrm{TiO}_{2}$ nanosheets with exposed (001) facets," Frontiers in Chemistry, vol. 6, p. 175, 2018.

[22] Y. Yang, J. Ye, L. Xu et al., "Synergistic effect of TNSs$\mathrm{TiO}_{2} \mathrm{NPs} / 3 \mathrm{DGN}$ catalysts on photocatalytic degradation of 4-nitrophenol under visible light," Applied Surface Science, vol. 433, pp. 398-407, 2018.

[23] M. L. Grilli, M. Yilmaz, S. Aydogan, and B. B. Cirak, "Room temperature deposition of XRD-amorphous $\mathrm{TiO}_{2}$ thin films: investigation of device performance as a function of temperature," Ceramics International, vol. 44, no. 10, pp. 1158211590, 2018.

[24] M. Shaban, A. M. Ashraf, and M. R. Abukhadra, "TiO ${ }_{2}$ nanoribbons/carbon nanotubes composite with enhanced photocatalytic activity; fabrication, characterization, and application," Scientific Reports, vol. 8, no. 1, p. 781, 2018.

[25] S. R. Sowmya, G. M. Madhu, and M. Hashir, "Studies on nanoengineered $\mathrm{TiO}_{2}$ Photo catalyst for effective degradation of dye," IOP Conference Series: Materials Science and Engineering, vol. 310, no. 1, article 012026, 2018.

[26] A. Casu, A. Lamberti, S. Stassi, and A. Falqui, "Crystallization of $\mathrm{TiO}_{2}$ nanotubes by in situ heating TEM," Nanomaterials, vol. 8, no. 1, p. 40, 2018.

[27] M. Ek, I. Beinik, A. Bruix, S. Wendt, J. V. Lauritsen, and S. Helveg, "Step edge structures on the anatase $\mathrm{TiO}_{2}(001)$ surface studied by atomic-resolution TEM and STM," Faraday Discussions, vol. 208, pp. 325-338, 2018.

[28] W. Zhang, J. Yang, and C. Li, "Role of thermal treatment on sol-gel preparation of porous cerium titanate: characterization and photocatalytic degradation of ofloxacin," Materials Science in Semiconductor Processing, vol. 85, pp. 33-39, 2018.

[29] S. R. Mirmasoomi, M. Mehdipour Ghazi, and M. Galedari, "Photocatalytic degradation of diazinon under visible light using $\mathrm{TiO}_{2} / \mathrm{Fe}_{2} \mathrm{O}_{3}$ nanocomposite synthesized by ultrasonicassisted impregnation method," Separation and Purification Technology, vol. 175, pp. 418-427, 2017.

[30] J. Y. Do, S. Choi, K. Nahm, S. K. Kim, and M. Kang, "Reliable hydrogen production from methanol photolysis in aqueous solution by a harmony between $\mathrm{In}$ and $\mathrm{Zn}$ in bimetallic zinc indium sulfide," Materials Research Bulletin, vol. 100, pp. 234-242, 2018.

[31] Y. Zhou, L. Zhang, and S. Tao, "Porous $\mathrm{TiO}_{2}$ with large surface area is an efficient catalyst carrier for the recovery of wastewater containing an ultrahigh concentration of dye," RSC Advances, vol. 8, no. 7, pp. 3433-3442, 2018.

[32] F. Qingge, C. Huidong, L. Haiying et al., "Synthesis and structural characteristics of high surface area $\mathrm{TiO}_{2}$ aerogels by ultrasonic-assisted sol-gel method," Nanotechnology, vol. 29, no. 7 , article $075702,2018$. 